\title{
On the Accuracy of High-Order Finite Elements in Curvilinear Coordinates
}

\author{
Stephen J. Thomas and Amik St.-Cyr \\ National Center for Atmospheric Research, \\ 1850 Table Mesa Drive, Boulder, 80305 CO, USA \\ \{thomas, amik\}@ucar.edu
}

\begin{abstract}
The governing equations for shallow water flow on the sphere are formulated in generalized curvilinear coordinates. The various analytic expressions for the differential operators are all mathematically equivalent. However, numerical approximations are not equally effective. The accuracy of high-order finite element discretizations are evaluated using the standard test problems proposed by Williamson et al (1992). The so-called strong conservation formulation is far more accurate and results in standard error metrics that are at least two orders of magnitude smaller than the weak conservation form, Jorgensen (2003), Prusa and Smolarkeiwicz (2003). Moreover, steady state solutions can be integrated much longer without filtering when time-stepping the physical velocities.
\end{abstract}

\section{Introduction}

The various terms that arise in a numerical model of the atmospheric general circulation (e.g. material derivative, gradient and divergence) have a tangible, physical existence that is independent of any coordinate-based description. However, coordinate-based representations are necessary for computing the explicit form of all requisite terms. Because the precise form of these terms depends upon the coordinate system being employed, a tensor representation is preferable. It allows the use of powerful mathematical tools to deduce relations that are valid in any coordinate system, i.e. coordinate invariant forms, while conveying the physical interpretation of the symbolic representation. For example, the equations of motion can be formulated using four different forms of the velocity; physical, contravariant, covariant or solenoidal. Although analytically equivalent, these lead to numerical approximations that are not equally effective. Here, we consider high-order finite element discretizations of the governing equations in curvilinear coordinates.

High-order finite elements are well-suited to atmospheric modeling due to their desirable numerical properties and inherent parallelism. The development of discontinuous Galerkin approximations can be viewed as an extension of low order finite-volume techniques for compressible flows with shocks (Cockburn et al 2000). Either nodal or modal basis functions can be employed in high-order 
finite elements which are spectrally accurate for smooth solutions. A discontinuous Galerkin approximation is equivalent to a conservative finite-volume discretization where the jump discontinuity at an element boundary requires the solution of a Riemann problem. In general, a filter is required to stabilize long time integrations of the nodal discontinuous approximation of the shallow water equations, due to the presence of nonlinear terms and their integration using inexact Gaussian quadrature, Giraldo et al (2003).

\section{Shallow Water Equations}

The shallow water equations contain the essential wave propagation mechanisms found in atmospheric general circulation models. These are the fast-moving gravity waves and nonlinear Rossby waves. The flux form shallow-water equations in curvilinear coordinates on the cubed-sphere are described in Rancic et al (1996) and Sadourny (1972). Covariant and contravariant vectors are related through the metric tensor by $u_{i}=G_{i j} u^{j}, u^{i}=G^{i j} u_{j}$, and $G=\left\{\operatorname{det}\left(G_{i j}\right)\right\}^{1 / 2}$.

$$
\begin{aligned}
\frac{\partial u_{1}}{\partial t} & =-\frac{\partial}{\partial x^{1}} E+G u^{2}(f+\zeta) \\
\frac{\partial u_{2}}{\partial t} & =-\frac{\partial}{\partial x^{2}} E-G u^{1}(f+\zeta) \\
\frac{\partial}{\partial t}(G \Phi) & =-\frac{\partial}{\partial x^{1}}\left(G u^{1} \Phi\right)-\frac{\partial}{\partial x^{2}}\left(G u^{2} \Phi\right)
\end{aligned}
$$

where

$$
E=\Phi+\frac{1}{2}\left(u_{1} u^{1}+u_{2} u^{2}\right), \quad \zeta=\frac{1}{G}\left[\frac{\partial u_{2}}{\partial x^{1}}-\frac{\partial u_{1}}{\partial x^{2}}\right] .
$$

$h$ is the height above sea level. $u^{i}$ and $u_{j}$ are the contravariant and covariant velocities. $\Phi=g h$ the geopotential height. $f$ is the Coriolis parameter.

The metric tensor for all six faces of the cube is

$$
G_{i j}=\frac{1}{r^{4} \cos ^{2} x^{1} \cos ^{2} x^{2}}\left[\begin{array}{cc}
1+\tan ^{2} x^{1} & -\tan x^{1} \tan x^{2} \\
-\tan x^{1} \tan x^{2} & 1+\tan ^{2} x^{2}
\end{array}\right]
$$

where $r=\left(1+\tan ^{2} x^{1}+\tan ^{2} x^{2}\right)^{1 / 2}$ and $G=1 / r^{3} \cos ^{2} x^{1} \cos ^{2} x^{2}$. A vector $\mathbf{v}=\left(v_{1}, v_{2}\right)$ in spherical coordinates is defined by its covariant and contravariant components. For the vector $\left(u^{1}, u^{2}\right)$ on the cube, the Jacobi matrix of the transformation between the sphere and cube is given by

$$
D=\left[\begin{array}{cc}
\cos \theta \partial \lambda / \partial x_{1} & \cos \theta \partial \lambda / \partial x_{2} \\
\partial \theta / \partial x_{1} & \partial \theta / \partial x_{2}
\end{array}\right]
$$

where $D^{T} D=G_{i j}$ and the cube to sphere mappings are

$$
D^{T}\left[\begin{array}{l}
v_{1} \\
v_{2}
\end{array}\right]=\left[\begin{array}{l}
u_{1} \\
u_{2}
\end{array}\right], \quad D\left[\begin{array}{l}
u^{1} \\
u^{2}
\end{array}\right]=\left[\begin{array}{l}
v_{1} \\
v_{2}
\end{array}\right] .
$$


The system of equations (11) is written in a weak conservation form. An alternative approach is to use a strong conservation form with physical velocities on the lhs of the momentum equation.

$$
\begin{aligned}
& \frac{\partial \mathbf{v}}{\partial t}=-(f+\zeta) \mathbf{k} \times \mathbf{v}-\frac{1}{2} \nabla(\mathbf{v} \cdot \mathbf{v})-\nabla \Phi \\
& \frac{\partial \Phi}{\partial t}=-\nabla \cdot \Phi \mathbf{v} .
\end{aligned}
$$

where

$$
\nabla \Phi=D^{-T}\left[\frac{\partial \Phi}{\partial x^{1}}, \frac{\partial \Phi}{\partial x^{2}}\right]^{T}, \quad \nabla \cdot \Phi \mathbf{v}=\frac{1}{G}\left[\frac{\partial G u_{1} \Phi}{\partial x^{1}}+\frac{\partial G u_{2} \Phi}{\partial x^{2}}\right]
$$

The kinetic energy is coordinate invariant and expressed in terms of spherical velocity components. The gradient is a covariant quantity and is mapped back to physical coordinates by the Jacobi matrix. The divergence is a scalar obtained by differentiating the contravariant velocity components. Taylor et al (1997) use an alternative approach where the time derivatives of the contravariant velocity components appear on the lhs of the momentum equation. Our numerical experiments clearly demonstrate that time integration of (2) leads to much smaller errors and reduces the amount of filtering required to stabilize the scheme.

\section{Space Discretization}

The computational domain $\Omega$ is partitioned into finite elements $\Omega_{k}$. An approximate solution $u_{h}$ belongs to the finite dimensional space $\mathcal{V}_{h}(\Omega) . u_{h}$ is expanded in terms of a tensor-product of the Lagrange basis functions defined at the Gauss-Lobatto-Legendre points

$$
u_{h}^{k}=\sum_{i=0}^{N} \sum_{j=0}^{N} u_{i j} h_{i}(x) h_{j}(y)
$$

A weak Galerkin variational problem is obtained by integrating the equations with respect to a test function $\varphi_{h} \in \mathcal{V}_{h}$. In the continuous Galerkin spectral element method, integrals are evaluated directly using Gauss-Lobatto quadrature

$$
\int_{\Omega_{k}} \phi_{h} u_{h} d \Omega=\sum_{i=0}^{N} \sum_{j=0}^{N} \phi_{h}\left(\xi_{i}, \xi_{j}\right) u_{h}\left(\xi_{i}, \xi_{j}\right) \rho_{i} \rho_{j}
$$

where $\left(\xi_{i}, \rho_{i}\right)$ are the Gauss-Lobatto nodes and weights. $C^{0}$ continuity is imposed in the spectral element method through the application of direct stiffness summation, Deville et al (2002).

To illustrate the discontinuous Galerkin approach, consider a scalar hyperbolic equation in flux form,

$$
u_{t}+\nabla \cdot \mathcal{F}=S
$$


By applying the Gauss divergence theorem, the weak form becomes

$$
\frac{d}{d t} \int_{\Omega_{k}} \varphi_{h} u_{h} d \Omega=\int_{\Omega_{k}} \varphi_{h} S d \Omega+\int_{\Omega_{k}} \mathcal{F} \cdot \nabla \varphi_{h} d \Omega-\int_{\partial \Omega_{k}} \varphi_{h} \mathcal{F} \cdot \hat{n} d s
$$

The jump discontinuity at an element boundary requires the solution of a Riemann problem where the flux function $\mathcal{F} \cdot \hat{n}$ is approximated by a Lax-Friedrichs numerical flux. The resulting semi-discrete equation is given by

$$
\frac{d u_{h}}{d t}=L\left(u_{h}\right)
$$

\section{$4 \quad$ Numerical Experiments}

Numerical experiments are based on of Williamson et al (1992). Test case 2 is a stationary zonal geostrophic flow. In theory, the equations can be integrated indefinitely with the only source of errors being due to numerical truncation. The continuous and discontinuous Galerkin spectral element models were integrated over 300 days using both the weak (1) and strong (2) conservation forms of the shallow water equations. The total number of elements was $9 \times 6=54$ and the number of Gauss-Lobatto-Legendre points per element was set to $16 \times 16$. In the case of the continuous Galerkin model, a second order explicit leap frog time integration scheme is applied together with a RGbert-Asselin (1972) time filter to damp the resulting computational mode. The time step size was $\Delta t=30 \mathrm{sec}$, respecting the CFL condition. The discontinuous Galerkin model is integrated with the second order three stage SSP Runge-Kutta scheme of Higueras (2004) using the same time step size. A spatial filter to stabilize the time step was not applied during any of these integrations, Fischer and Mullen (2001). Figure 1 contains plots of the eigenvalues of the inverse metric tensor $G^{i j}$ used to map covariant to contravariant quantities on the cube. These clearly illustrate the magnitude of the stretching across one face of the cube-sphere.

The results of the Centinuous Galerkin model integrations reveal that the weak formulation becomes unstable after only six days. Whereas the strong form can be stably integrated for over 300 days without any spatial filtering with a small growth in the error level. We attribute these results to several factors. Weak conservative formulations implicitly map between the sphere and cube with the inverse metric tensor, whereas the strong form relies on the Jacobi matrix. The eigenvalues of the latter are a factor of $\sqrt{2}$ smaller. Figure 2 is a plot of the $l_{\infty}$ geopotential height errors for the continuous Galerkin formulations. These plots show that the errors are two orders of magnitude smaller for the strong conservation formulation. The results for the discontinuous Galerkin scheme are similar, namely the weak formulation becomes unstable after six days of integration. Once again the strong form remains stable for well over 300 days of integration. Furthermore, the scheme conserves mass and the $l_{\infty}$ error remains close to machine precision. 

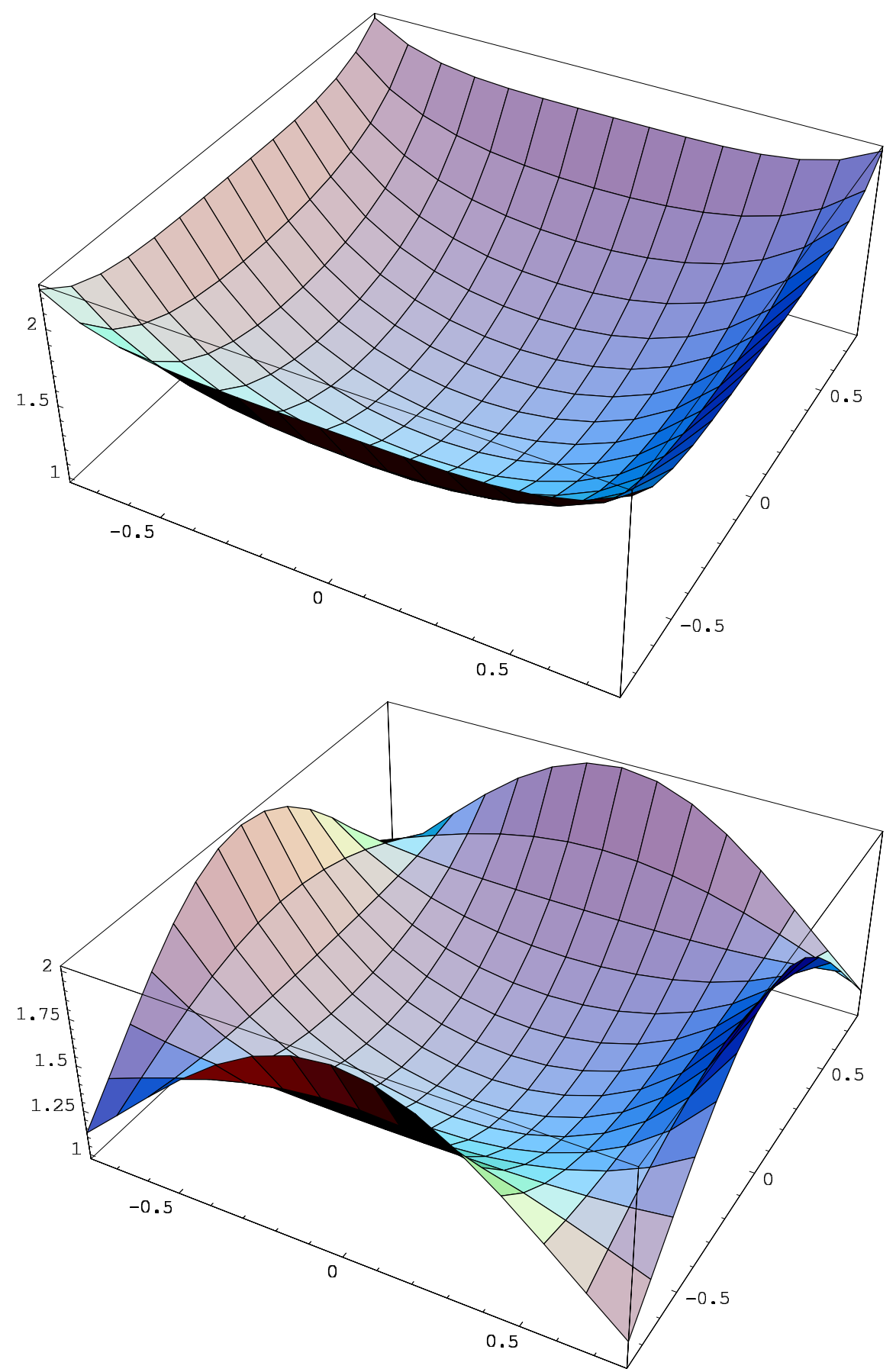

Fig. 1. Eigenvalues of the inverse metric tensor $G^{i j}$ used to map covariant to contravariant quantities on the cube. Top: $\lambda_{1}$. Bottom: $\lambda_{2}$ 

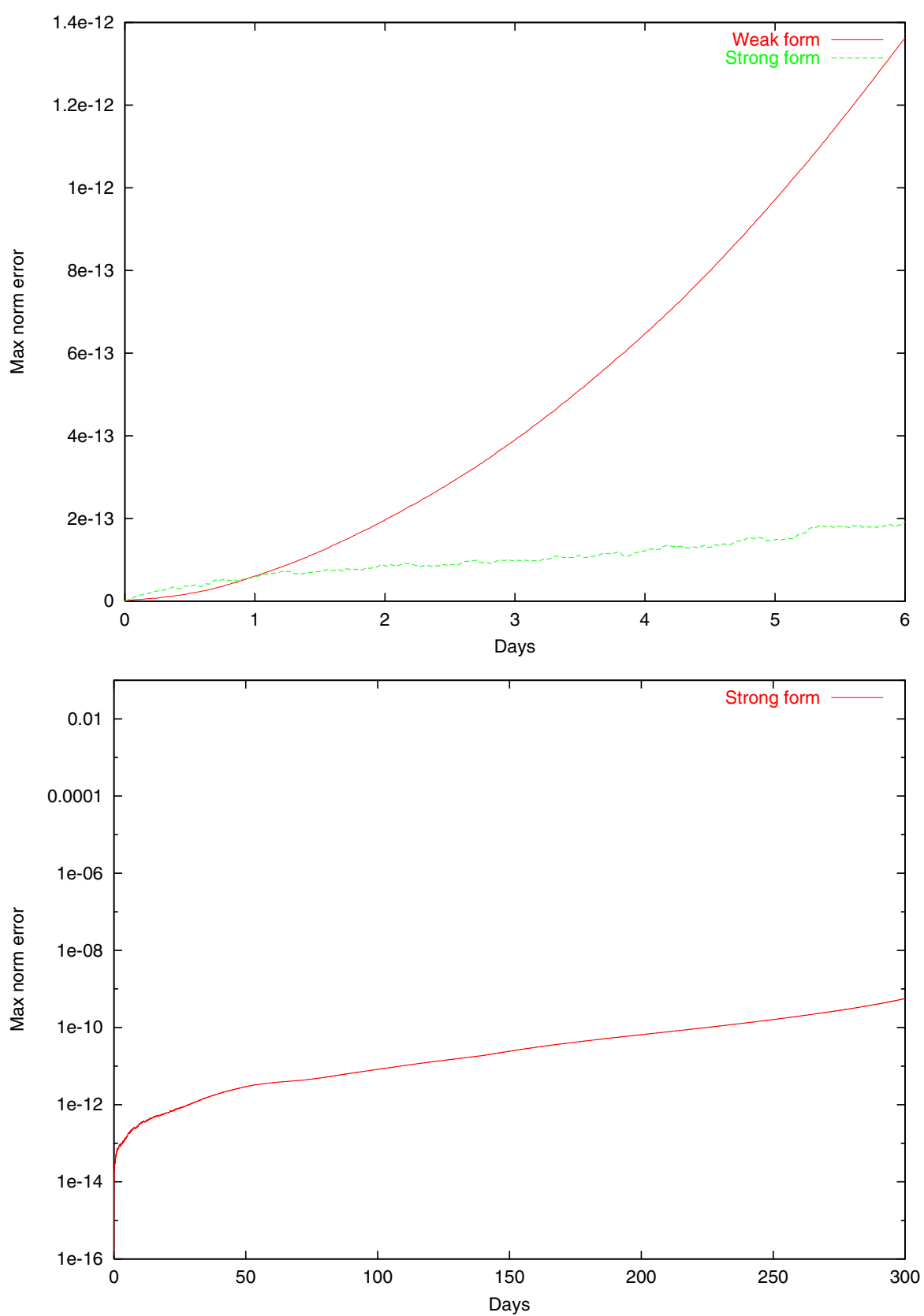

Fig. 2. Shallow water test case 2: Stationary geostrophic flow. $9 \times 6=54$ continuous Galerkin spectral elements. $16 \times 16$ Gauss-Lobatto-Legendre points per element. $\Delta t=$ $30 \mathrm{sec}$. No spatial filter applied. Top: $l_{\infty}$ error for weak and strong conservation forms integrated to 6 days. Bottom: $l_{\infty}$ for strong conservation form integrated to 300 days 


\section{Conclusions}

Taylor et al (1997) were not able to achieve or maintain machine precision level errors because they employed a weak conservation form for the governing equations of motion. To fully exploit the accuracy of high-order finite elements, a judicious choice of an appropriate form of the prognostic equations is required when using generalized curvilinear coordinates. Our experience with a nodal Galerkin method indicates that a filter is ultimately required for long integrations to stabilize the scheme, thereby improving and extending the recent results of Nair et al (2004).

\section{References}

1. Asselin, R., 1972: Frequency filter for time integrations. Mon. Wea. Rev., 100, 487-490.

2. Cockburn, B., G. E. Karniadakis, and C. W. Shu, 2000: Discontinuous Galerkin Methods. Springer-Verlag, New York, 470 pp.

3. Deville, M. O., P. F. Fischer, and E. H. Mund, 2002: High-Order Methods for Incompressible Fluid Flow. Cambridge University Press, 499 pp.

4. Fischer, P. F., and J. S. Mullen, 2001: Filter-Based stabilization of spectral element methods. Comptes Rendus de l'Académie des sciences Paris, t. 332, Série I Analyse numérique, 265-270.

5. Giraldo, F. X., J. S. Hesthaven, and T. Warburton, 2003: Nodal high-order discontinuous Galerkin methods for spherical shallow water equations. J. Comput. Phys., 181, 499-525.

6. Higueras, I., 2004: On strong stability preserving time discretization methods. $J$. Sci. Comput., 21, 193-223.

7. Jorgensen, B. H. , 2003: Tensor formulations of the model equations in strong conservation form for an incompressible flow in general coordinates Technical Report Riso-R-1445, Riso National Laboratory, Roskilde, Denmark.

8. Nair, R. D., S. J. Thomas, and R. D. Loft, 2004: A discontinuous Galerkin global shallow water model. Mon. Wea. Rev., to appear.

9. Prusa, J. M. , and P. K. Smolarkiewicz, 2003: An all-scale anelastic model for geophysical flows: Dynamic grid deformation. J. Comp. Phys., 190, 601-622.

10. Rancic, M., R. J. Purser, and F. Mesinger, 1996: A global shallow-water model using an expanded spherical cube: Gnomic versus conformal coordinates. $Q . J . R$. Meteorol. Soc., 122, 959-982.

11. Sadourny, R., 1972: Conservative finite-difference approximations of the primitive equations on quasi-uniform spherical grids. Mon. Wea. Rev., 100, 136-144.

12. Taylor, M., J. Tribbia, M. Iskandarani, 1997: The spectral element method for the shallow water equations on the sphere. J. Comp. Phys., 130, 92-108.

13. Williamson, D. L., J. B. Drake, J. J. Hack, R. Jakob, P. N. Swarztrauber, 1992: A standard test set for numerical approximations to the shallow water equations in spherical geometry J. Comp. Phys., 102, 211-224. 